\title{
A Direct Measurement of Atmospheric Dispersion in N-band Spectra: Implications for Mid-IR Systems on ELTs ${ }^{\star}$
}

\author{
Andrew J. Skemer ${ }^{1, \text { a }}$, Philip M. Hinz ${ }^{1}$, William F. Hoffmann ${ }^{1}$, Laird M. Close ${ }^{1}$, Sarah Kendrew ${ }^{2}$, \\ Richard J. Mathar ${ }^{2}$, Remko Stuik ${ }^{2}$, Thomas P. Greene ${ }^{3}$, Charles E. Woodward ${ }^{4}$, and Michael S. \\ Kelley $^{5}$ \\ 1 Steward Observatory, Department of Astronomy, University of Arizona, Tucson, AZ 85721 \\ 2 Leiden Observatory, Leiden University, The Netherlands \\ 3 NASA Ames Research Center, Moffett Field, CA 94035 \\ 4 Department of Astronomy, School of Physics and Astronomy, University of Minnesota, Minneapo- \\ lis, MN 55455 \\ 5 Department of Astronomy, University of Maryland, College Park, MD 20742-2421
}

\begin{abstract}
This paper is modified from [1]. Adaptive optics will almost completely remove the effects of atmospheric turbulence at $10 \mu \mathrm{m}$ on the Extremely Large Telescope (ELT) generation of telescopes. In this paper, we observationally confirm that the next most important limitation to image quality is atmospheric dispersion, rather than telescope diffraction. By using the 6.5 meter MMT with its unique mid-IR adaptive optics system, we measure atmospheric dispersion in the N-band with the newly commissioned spectroscopic mode on MIRAC4BLINC. Our results indicate that atmospheric dispersion is generally linear in the N-band, although there is some residual curvature. We compare our measurements to theory, and make predictions for ELT Strehls and image FHWM with and without an atmospheric dispersion corrector (ADC). We find that for many mid-IR applications, an ADC will be necessary on ELTs.
\end{abstract}

\section{Introduction}

As we approach the Extremely Large Telescope (ELT) generation of telescopes, adaptive optics is becoming increasingly important to the general astronomical community. Large telescopes have small diffraction limits, and achieving these limits is a major goal for instrument builders. The mid-infrared wavelengths, in particular, stand to gain substantially from larger telescopes-at the diffraction limit, $\mathrm{S} / \mathrm{N} \propto$ Diameter $^{2}$ for background-limited observations of point-sources. Today's $\sim 8$-meter class telescopes are close to diffraction-limited in the mid-infrared, even without adaptive optics, but maintaining the diffraction limit as telescopes continue to scale upwards will be challenging.

For ground-based telescopes in the mid-infrared, seeing is often considered a minor effect, and other (smaller) atmospheric effects are completely ignored. [2] have predicted several atmospheric properties that may limit image quality on ELTs, including mid-infrared atmospheric dispersion ${ }^{1}$, visible atmospheric dispersion for wavefront sensing, and water vapor turbulence. So far, these effects have not been adequately measured.

The 6.5 meter MMT, with its unique mid-IR adaptive optics system (MMTAO) provides a powerful testbed for mid-IR AO on ELTs. Effects that will severely limit image quality on ELTs are just measurable with MMTAO due to its highly stable PSF. By removing the largest atmospheric effect (seeing), we measure the second largest effect (atmospheric dispersion) with the newly commissioned

\footnotetext{
* The observations reported here were obtained at the MMT Observatory, a facility operated jointly by the Smithsonian Institution and the University of Arizona

a e-mail: askemer@as.arizona.edu

${ }^{1}$ In this paper, we use "refraction" to refer to the absolute bending of light and "dispersion" to refer to the differential chromatic bending of light. Generally, theoretical considerations use refraction while practically, telescope images are affected by dispersion.
}

This is an Open Access article distributed under the terms of the Creative Commons Attribution-Noncommercial License, which permits unrestricted use, distribution, and reproduction in any noncommercial medium, provided the original work is properly cited. 
spectroscopic mode of the MMT's Mid-Infrared Array Camera (MIRAC4-BLINC) and adaptive optics.

Atmospheric refraction is a well-known phenomenon at visible wavelengths, where it is typically treated as a smooth curve that flattens quickly longward of K-band [3-5]. However, more detailed treatments show that molecular resonances from $\mathrm{CO}_{2}$ and $\mathrm{H}_{2} \mathrm{O}$ (amongst others) dominate the infrared refractivity curve [6-9]. These authors show that each infrared window $(\mathrm{L}, \mathrm{M}, \mathrm{N})$ is bracketed by molecular absorption and has an atmospheric refraction curve characterized by an S-shape superimposed on a stronger linear trend.

In this paper, we measure the atmospheric dispersion curve on the short wavelength side of N-band $(8.26 \mu \mathrm{m}-11.27 \mu \mathrm{m})$ using spectroscopy and adaptive optics. In previous studies, [10] measured refractivity at one wavelength $(12 \mu \mathrm{m})$ while [11] interferometrically measured refractivity throughout the $\mathrm{N}$-band but were insensitive to the overall trend. Our spectroscopic result has the benefit of measuring all wavelengths simultaneously, so that the overall trend and curvature of the effect throughout N-band is unambiguous. By directly measuring the atmospheric dispersion curve, we can assess how the effect will limit image quality in the mid-infrared for ground-based ELTs. This is useful for instrument builders, who will have the option of using atmospheric dispersion correctors (ADCs) to suppress the effect.

\section{Observations and Analysis}

Our data were obtained March 4, 2009 UT with the 6.5 meter MMT and its deformable secondary adaptive optics system (MMTAO [12-14]). We used the newly commissioned spectroscopic mode of MIRAC4-BLINC. The instrument is a combination of the Mid-IR Array Camera, Gen. 4 (MIRAC4) and the Bracewell Infrared Nulling Cryostat (BLINC [15]) which for these observations, is used in its "imaging" mode. MIRAC4 is functionally similar to previous incarnations of MIRAC [16] with the main new feature being a DRS Technologies 256 x $256 \mathrm{Si}$ :As array. A description of the instrument can be found in [1]. An example of the MMTAO/MIRAC4-BLINC PSF, taken at $9.15 \mu \mathrm{m}$, is shown in Figure 1.

To measure atmospheric dispersion, we took spectra of standards at a variety of airmasses, and determined how the grism trace (centroid of the spectrum as a function of wavelength) varied with airmass. By using our lowest airmass observation (1.05 airmasses) as a standard for intrinsic grism curvature, we were able to measure the excess curvature as a function of airmass $(1.32,1.53,1.82$, and 2.53 airmasses), which is a direct measurement of atmospheric dispersion. The models of [7,9] suggest that the effect of atmospheric dispersion at 1.05 airmasses is small, but non-negligable. As

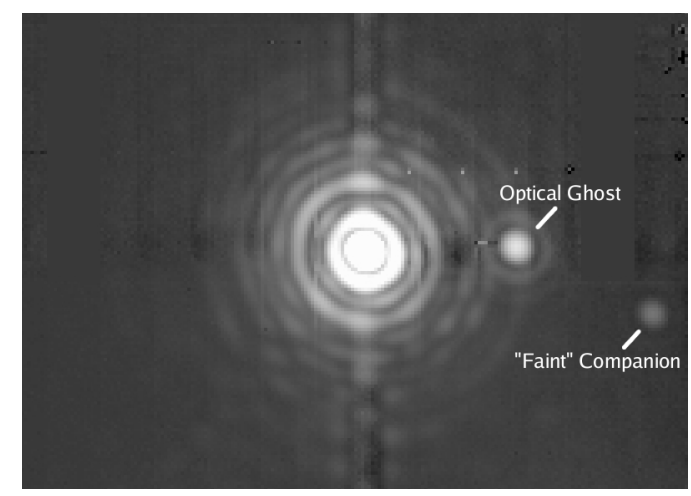

Fig. 1. An example of MMTAO/MIRAC4-BLINC's imaging capabilities at $9.15 \mu \mathrm{m}$ (single 60 s frame with the adaptive optics system running at $550 \mathrm{~Hz}$ ). The primary star is $\alpha$ Her, and the system creates an optical ghost to the right. To the lower right is the "faint" companion, $\alpha$ Her B. The image is 7.8" x 11" and is displayed with a logarithmic stretch. 


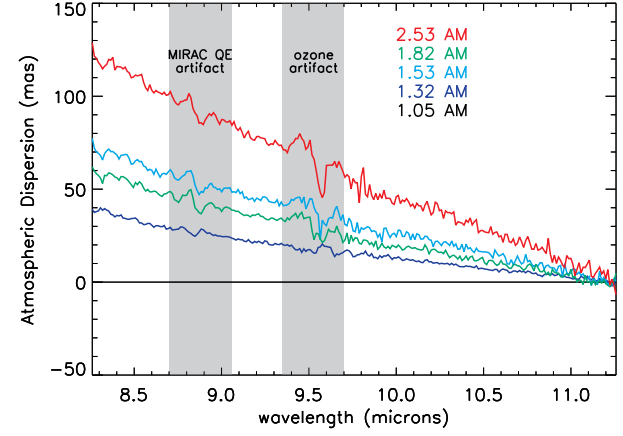

(a)

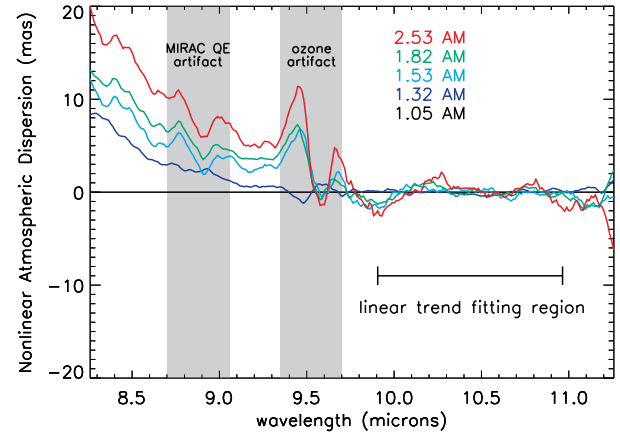

(b)

Fig. 2. Figure from [1]. (a) MIRAC4-BLINC/MMTAO measurements of atmospheric dispersion in the N-band. For each airmass, we measure the grism trace and subtract the grism's intrinsic curvature. The effect has been fixed to 0 at $11.2 \mu \mathrm{m}$. Our results show that atmospheric dispersion in the mid-infrared is a relatively large effect, although it is considerably smaller than the $\sim 0.3$ " diffraction-limited FWHM of a 6.5 meter telescope. On larger ELTs, mid-IR atmospheric dispersion will severely limit image quality if left uncorrected. Note that we underestimate atmospheric dispersion by a small amount (15 mas cumulative) due to our assumption that atmospheric dispersion is negligible at 1.05 airmasses. (b) On ELTs, mid-IR atmospheric dispersion correctors (ADCs) will be necessary to suppress the linear atmospheric refraction effect shown in (a). In (b), we fit and remove the linear trend from each atmospheric dispersion curve. The resultant nonlinear residuals will be uncorrected by traditional ADCs.

a result, all of our measurements underestimate the effect of atmospheric dispersion by the amount shown in Figure 3 of [1] at each airmass (a cumulative effect of 0.015").

The effect is fixed to 0 at the red end $(11.2 \mu \mathrm{m})$ of our spectrum and is shown for each observed airmass in Figure 2a. There is clear evidence that the blue light is refracted more than the red, and that this trend increases with airmass. A fit of the linear trend between 9.9 and $11.0 \mu \mathrm{m}$ gives $10.3 \mathrm{mas} / \mu \mathrm{m}$, $20.4 \mathrm{mas} / \mu \mathrm{m}, 15.8 \mathrm{mas} / \mu \mathrm{m}$ and $33.9 \mathrm{mas} / \mu \mathrm{m}$ at 1.32 airmasses, 1.53 airmasses, 1.82 airmasses and 2.53 airmasses respectively. We note that the observed trend is not perfectly sequential as our 1.53 airmass data appear to have experienced more atmospheric dispersion than our 1.82 airmass data.

The strong linear trends from Figure 2a imply that atmospheric dispersion is an important effect to consider when designing mid-IR instruments on large telescopes. For the next (ELT) generation of telescopes, a mid-IR ADC will be necessary to achieve diffraction-limited images. However, traditional ADCs can only correct linear atmospheric dispersion. In Figure $2 b$ we show the curvature of atmospheric dispersion by subtracting off a linear trend from the data shown in Figure 2a. This simulates the effect of atmospheric dispersion after correction from a perfectly tuned ADC. Unfortunately, some elongation of the PSF may still occur in broad filters even with an ADC due to nonlinear atmospheric dispersion. Note that the observed curvature is sequential with airmass.

\section{Comparison with Models}

We compare our measurements to the models described by [7,9]. These models calculate refractive index values $(n)$ by summing over the electronic transitions of atmospheric molecular constituents from the far-ultraviolet to the far-infrared, using the molecular line database HITRAN [17]. A full description of the model can be found in [7].

The comparison between our measurements and the models is shown in Figure 3. The solid curves are our measurements (with atmospheric dispersion at 1.05 airmasses subtracted), the dotted curves are the models with dispersion at 1.05 airmasses subtracted and the dashed curves are the models without dispersion at 1.05 airmasses subtracted. 
The models show a good fit to the observed spectral trace longward of $\sim 9.5 \mu \mathrm{m}$ in three of the four cases. The clear exception is the spectral trace taken at airmass 1.53 , which follows the same trend as the others but lies significantly above the curve predicted for the meteorological conditions at the time of observation. At all four airmasses, our measurements show more curvature than the models predict. At this point, it is unclear whether the source of disagreement is error in the measurements or error in the models. Given that the linear trend dominates the curvature, this disagreement may prove insignificant. However, if the linear trend is corrected with an ADC, the curvature may still cause some non-negligble dispersion at very high airmasses.
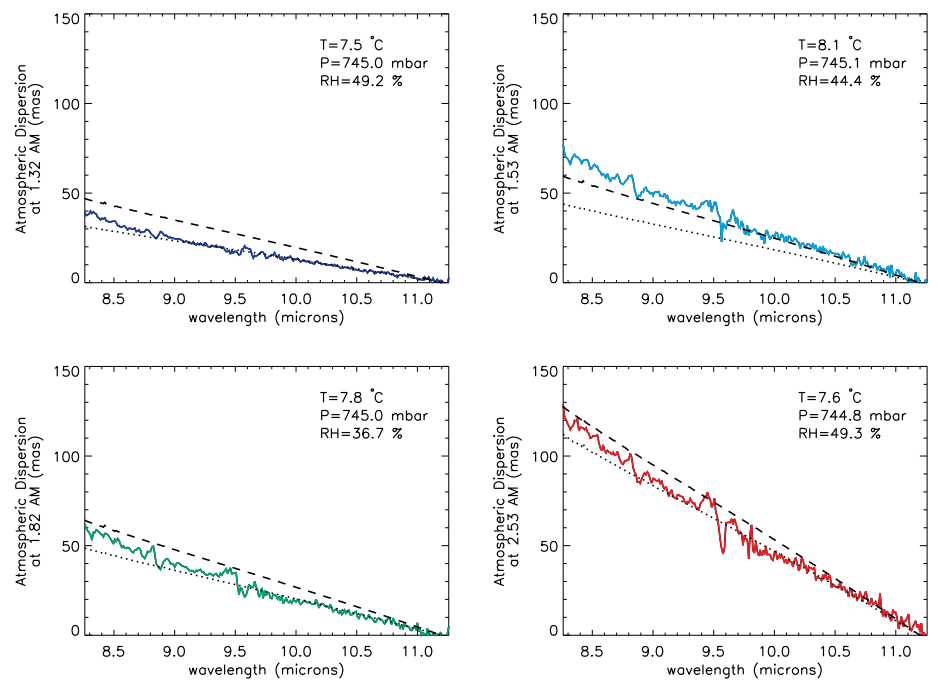

Fig. 3. Figure from [1]. Comparisons of our measured atmospheric dispersion with theory from [7,9]. Note that we underestimate atmospheric dispersion by a small amount due to our assumption that atmospheric dispersion is negligible at 1.05 airmasses. In all four plots, the solid, colored curves are our measurements, the dotted curves are models that subtract the 1.05 airmass dispersion to properly account for the underestimate described above, and the dashed curves are the true models. The models all have been calculated using weather data from the corresponding observation, and have errors of about $10 \%$ based on the varying humidity during each observation. Three of the four models are very good fits to the linear trend of our measurements. However, the models all indicate less curvature than is seen in the data.

\section{Implications for ELTs}

Using our MMTAO observations, we can simulate the degradation of image quality for ELTs. Our MMTAO observations only cover $8.26 \mu \mathrm{m}-11.27 \mu \mathrm{m}$ but the broad N-band extends all the way to $\sim 14 \mu \mathrm{m}$. We approximate full $\mathrm{N}$-band dispersion curves $(8.26 \mu \mathrm{m}-13.74 \mu \mathrm{m})$ by reflecting our MMTAO dispersion curves about $10.5 \mu \mathrm{m}$ (creating the characteristic S-shape; this may be an oversimplification as [7,9]'s models show slightly increased curvature longward of $11 \mu \mathrm{m}$ ). We also add back the theoretical dispersion at 1.05 airmasses having confirmed the validity of the linear trends in [7,9]'s models. Finally, we fit the curves with a fifth-order polynomial to remove the noise and systematics shown in Figure 2.

We simulate ELT images by convolving our estimated dispersion curves with diffraction-limited PSFs and flat SEDs. The PSFs are constructed from annulus apertures with outer diameters $24.5 \mathrm{~m}$, $30 \mathrm{~m}$ and $42 \mathrm{~m}$ and a $20 \%$ central obscuration. An example of our simulated images is shown for a 42 meter telescope with no ADC in Figure 4. The results show a severe elongation in the altitude axis. 


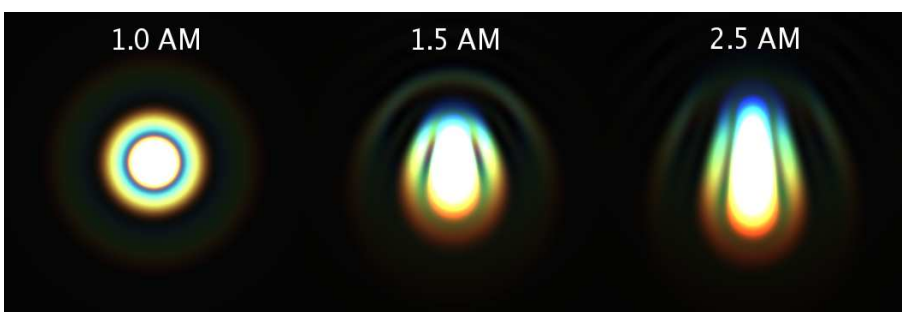

Fig. 4. Figure from [1]. Simulated 3-color N-band images (blue is $8.26 \mu \mathrm{m}$ and red is $13.74 \mu \mathrm{m}$ ) for a 42 meter telescope at different airmasses (zenith is up). Without an ADC, image quality will be significantly degraded in the altitude axis. The images assume a flat SED and a site similar to the MMTs.

Using our simulated ELT images, we measure Strehl and FWHM, with and without a linear ADC, at 1.0, 1.5 and 2.5 airmasses. These results are shown in Table 1. Without a linear ADC, Strehl and FWHM are significantly degraded for all three telescopes, even at 1.5 airmasses. With a linear ADC, the images are almost perfectly corrected at reasonable airmasses. The experiment is repeated for a $10 \%$ filter in Table 2.

Table 1. Predicted N-band Image Quality for ELTs

\begin{tabular}{cccccc}
\hline Telescope Diameter $(\mathrm{m})$ & Airmass & Strehl $(\%)$ & Strehl with ADC (\%) & FWHM (mas) & FWHM with ADC (mas) \\
\hline 42 & 1.0 & 100 & 100 & 53 & 53 \\
42 & 1.5 & 43 & 98 & 135 & 54 \\
42 & 2.5 & 27 & 94 & 226 & 56 \\
\hline 30 & 1.0 & 100 & 100 & 74 & 74 \\
30 & 1.5 & 56 & 99 & 139 & 75 \\
30 & 2.5 & 37 & 97 & 225 & 91 \\
24.5 & 1.0 & 100 & 100 & 91 & 92 \\
24.5 & 1.5 & 64 & 99 & 225 & 93 \\
\hline 2.5 & 2.5 & 44 & 98 & & \\
\hline
\end{tabular}

The N-band filter is assumed to be rectangular from $8.26 \mu \mathrm{m}-13.74 \mu \mathrm{m}$. We also assume a flat SED and a site similar to the MMT's. FWHM is measured in the altitude axis. In the azimuth axis, FWHM is assumed to be diffraction-limited.

Table 2. Predicted 10\%-band Image Quality for ELTs

\begin{tabular}{cccccc}
\hline Telescope Diameter $(\mathrm{m})$ & Airmass & Strehl $(\%)$ & Strehl with ADC (\%) & FWHM (mas) & FWHM with ADC (mas) \\
\hline 42 & 1.0 & 100 & 100 & 52 & 52 \\
42 & 1.5 & 94 & 100 & 55 & 52 \\
42 & 2.5 & 88 & 100 & 58 & 52 \\
\hline 30 & 1.0 & 100 & 100 & 73 & 73 \\
30 & 1.5 & 97 & 100 & 74 & 73 \\
30 & 2.5 & 93 & 100 & 77 & 89 \\
24.5 & 1.0 & 100 & 100 & 89 & 89 \\
24.5 & 1.5 & 98 & 100 & 90 & 89
\end{tabular}

The $10 \%$-band filter is assumed to be rectangular from $9.975 \mu \mathrm{m}-11.025 \mu \mathrm{m}$. We also assume a flat SED and a site similar to the MMT's. FWHM is measured in the altitude axis. In the azimuth axis, FWHM is assumed to be diffraction-limited. 


\section{Conclusions}

After seeing is removed by adaptive optics, atmospheric dispersion will be the dominant source of image quality degradation on ground-based ELTs, surpassing diffraction. While theory has predicted large S-shaped refraction curves in each infrared window, the effect has not been measured as a function of wavelength through the atmosphere. In this paper, we use MMTAO and the MIRAC4-BLINC spectrograph to measure atmospheric dispersion from $8.26 \mu \mathrm{m}-11.27 \mu \mathrm{m}$. We find the following:

1) "Blue"-light $(8.26 \mu \mathrm{m})$ is refracted more than "red"-light $(11.27 \mu \mathrm{m})$ in the mid-infrared, and the effect increases with airmass and humidity.

2) Although the linear term of mid-IR atmospheric dispersion is dominant, a non-negligable amount of curvature may exist too. Observations over different wavelength ranges (including full N-band) will help determine the source of this curvature.

3) The dominating linear trend in our measurements are in excellent agreement with the models of $[7,9]$. We measure more curvature than the theory predicts; however, the magnitude of the curvature is small compared to the linear trend. Models from [7,9] may now be used to develop predictive models for ADCs given ground-based measurements of temperature, pressure, relative humidity and airmass.

4) Based on simulations of mid-IR ELT adaptive optics images with atmospheric dispersion, we find that ADCs will be useful for high-Strehl, narrow-band imaging and spectroscopy, and essential for high-Strehl, broad-band imaging and spectroscopy. Our conclusions are only based on an analysis of image quality. We make no claims about the technical feasibility (cost, increased background, decreased throughput, etc.) of a mid-IR ADC. Instrument builders will have to weigh these issues as well.

\section{References}

1. A.J. Skemer, P.M. Hinz, W.F. Hoffmann, L.M. Close, S. Kendrew, R.J. Mathar, R. Stuik, T.P. Greene, C.E. Woodward, M.S. Kelley, PASP 121, 897 (2009)

2. S. Kendrew, L. Jolissaint, R.J. Mathar, R. Stuik, S. Hippler, B. Brandl, SPIE 7015 (2008)

3. B. Edlén, Metrologia 2, 71 (1966)

4. P.E. Ciddor, Applied Optics 35, 1566 (1996)

5. G. Bönsch, E. Potulski, Metrologia 35, 133 (1998)

6. R.J. Hill, R.S. Lawrence, Infrared Physics 26, 371 (1986)

7. R.J. Mathar, Applied Optics 43, 928 (2004)

8. M.M. Colavita, M.R. Swain, R.L. Akeson, C.D. Koresko, R.J. Hill, PASP 116, 876 (2004)

9. R.J. Mathar, Journal of Optics A: Pure and Applied Optics 9, 470 (2007)

10. T.A. Livengood, K.E. Fast, T. Kostiuk, F. Espenak, D. Buhl, J.J. Goldstein, T. Hewagama, K.H. Ro, PASP 111, 512 (1999)

11. R.N. Tubbs, J.A. Meisner, E.J. Bakker, S. Albrecht, SPIE 5491, 588 (2004)

12. M. Lloyd-Hart, PASP 112, 264 (2000)

13. F.P. Wildi, G. Brusa, M. Lloyd-Hart, L.M. Close, A. Riccardi, SPIE 5169, 17 (2003)

14. G. Brusa, D.L. Miller, M.A. Kenworthy, D.L. Fisher, A. Riccardi, SPIE 5490, 23 (2004)

15. P.M. Hinz, J.R.P. Angel, N.J. Woolf, W.F. Hoffmann, D.W. McCarthy, SPIE 4006, 349 (2000)

16. W.F. Hoffmann, J.L. Hora, G.G. Fazio, L.K. Deutsch, A. Dayal, SPIE 3354, 647 (1998)

17. L.S. Rothman, D. Jacquemart, A. Barbe, D. Chris Benner, M. Birk, L.R. Brown, M.R. Carleer, C. Chackerian, K. Chance, L.H. Coudert et al., Journal of Quantitative Spectroscopy and Radiative Transfer 96, 139 (2005) 